\title{
Use of Choquet integrals in multivalued contexts
}

\author{
Cristina Alcalde • Ana Burusco
}

Received: date / Accepted: date

\begin{abstract}
In this work, multivalued contexts are studied, in which the different observations are related to each other. Continuing the studies already carried out in previous works, the use of Choquet integrals can be an adequate tool for this situations. If in addition the set of objects or attributes of these contexts represents a temporal sequence, we can also represent these contexts as sequences of contexts that evolve in time and we can use tools in this field to extract information. Finally, as illustrative application, the developed theory is used to measure student progress in learning.
\end{abstract}

Keywords $L$-fuzzy concept analysis, $L$-fuzzy context sequences, WOWA operators, Choquet integrals

\section{Introduction}

The Formal Concept Analysis [34,24] extracts information from a binary table that represents a formal context $(X, Y, R)$ with $X$ and $Y$ finite sets of objects and attributes respectively and $R \subseteq X \times Y$. The hidden information consists of pairs $(\bar{A}, B)$ with $A \subseteq X$ and $B \subseteq Y$, called formal concepts, verifying $A^{*}=B$ and

\section{Alcalde}

Dept. Applied Mathematics

University of the Basque Country - UPV/EHU

Plaza de Europa 1, 20018 San Sebastian, Spain

E-mail: c.alcalde@ehu.es

A. Burusco

Departament of Statistics, Computer Science and Mathematics

Public University of Navarre

Campus de Arrosadía, 31006 Pamplona, Spain

Institute of Smart Cities

Campus de Arrosadía, 31006 Pamplona, Spain

E-mail: burusco@unavarra.es
$B^{*}=A$, where $(\cdot)^{*}$ is the derivation operator that associates the attributes related to the elements of $A$ to every object set $A$, and the objects related to the attributes of $B$ to every attribute set $B$. These formal concepts can be interpreted as a group of objects $A$ that shares the attributes of $B$.

In previous works $[13,15]$ we have defined the $L$ fuzzy contexts $(L, X, Y, R)$, with $L$ a complete lattice, $X$ and $Y$ sets of objects and attributes respectively and $R \in L^{X \times Y}$ a fuzzy relation between the objects and the attributes. This is an extension of the formal contexts of Wille to the fuzzy case when we want to study the relations between the objects and the attributes with values in a complete lattice $L$, instead of binary ones. The $L$-fuzzy concept analysis has been developed as a tool for the extraction of knowledge from these $L$-fuzzy contexts using $L$-fuzzy concepts.

These fuzzy techniques have shown to be very useful in other areas of work as in $[9,10,29,8]$.

In the past [14] we also studied the $L$-fuzzy contexts where we have several values representing the relation between every object and every attribute. In that case the multisets were used as a tool for working in that situation without the need of aggregated observations.

Later [17], we analyzed the contexts with multiple weighted values where the set of experts $E$ and weights $P$ were introduced.

The reason why we have considered interesting to analyze this topic is that, in many situations, the relationship between the objects and the attributes of a context may not be unique. Let us think, for instance, of a group of experts giving their opinion about the influence of some symptoms in a group of diseases. On the other hand, we are aware that, due to the large volume of data, sometimes the aggregation of observations may be the optimal way of working. It will be 
extremely important to know where these observations come from and to take into account the relationship existing among them.

The election of a good aggregation operator can be crucial to obtain a more complete information. Recently [5], WOWA operators have been used as aggregation tools in these contexts that represent the opinion of multiple experts. However, it is important to be open to new aggregation tools, such as the integral Choquet [21]. This aggregation method will allow a more complete analysis of the proposed problem. In this paper, we will see that the results obtained in previous works can be improved using these Choquet integrals when there exist dependencies among the sources of the data.

Besides, these multivalued contexts can be interpreted as sequences of $L$-fuzzy contexts, and hence the use of other tools associated with sequences of contexts can be a good option in order to obtain a more complete analysis of the data. These tools are techniques that can be used for extract an underline patterns of behavior that provide a base for making decisions.

In the next section we will begin recovering the most important results about aggregation operators and $L$ fuzzy concept analysis that will be useful in the rest of the work.

\section{Preliminaries}

In the paper, different aggregation operators are used depending on the nature of the data to be aggregated.

\subsection{Aggregation operators}

Families of OWA operators were introduced by Yager [35] as a new aggregation technique based on the ordered weighted averaging. The definition of these operators is as follows.

Definition 1 [35] A mapping $F_{w}$ from $L^{n} \longrightarrow L$, where $L=[0,1]$ is called an OWA operator of dimension $n$ if associated with $F_{w}$ is a weighting $n$-tuple $w=$ $\left(w_{1}, w_{2}, \ldots, w_{n}\right)$ such that $w_{i} \in[0,1]$ and $\sum_{1 \leq i \leq n} w_{i}=$ 1 , where $F_{w}\left(a_{1}, a_{2}, \ldots, a_{n}\right)=w_{1} \cdot b_{1}+w_{2} \cdot b_{2}+\cdots+$ $w_{n} . b_{n}$, with $b_{i}$ the $i$ th largest element in the collection $a_{1}, a_{2}, \ldots, a_{n}$.

We applied these OWA operators to the fuzzy contexts sequences to analyze tendencies when the sequence represents the evolution in time [7].

After that, the Weighted OWA operators (WOWA) were defined [32] and combine the advantages of the OWA operators and the ones of the weighted mean $[19$,
20]. These operators consider two weighting vectors: $w=\left(w_{1}, w_{2}, \ldots, w_{n}\right)$ corresponding to the relevance of the values (operator OWA) and $p=\left(p_{1}, p_{2}, \ldots, p_{n}\right)$ corresponding to the relevance of the sources or experts.

As particular cases, if $w_{i}=1 / n, \forall i$ then we have the weighted mean with $p$ and if $p_{i}=1 / n, i \in\{1, \ldots, n\}$, an OWA operator with $w$.

This is the definition:

Definition 2 [32] Let $p, w$ be weighting vectors of dimension $n, p=\left(p_{1}, p_{2}, \ldots, p_{n}\right)$ and $w=\left(w_{1}, w_{2}, \ldots, w_{n}\right)$ such that $p_{i}, w_{i} \in[0,1]$ and $\sum_{i} p_{i}=\sum_{i} w_{i}=1$.

In this case, a mapping $F_{p w}^{i}: \mathbb{R}^{n} \stackrel{i}{\longrightarrow} \mathbb{R}$ is a Weighted Ordered Weighted Averaging (WOWA) operator of dimension $n$ if

$F_{p w}\left(a_{1}, a_{2}, \ldots, a_{n}\right)=\sum_{i} \omega_{i} a_{\sigma(i)}$

where $\{\sigma(1), \ldots, \sigma(n)\}$ is a permutation of $\{1, \ldots, n\}$ such that $a_{\sigma(i-1)} \geq a_{\sigma(i)}$ for all $i \in\{2, \ldots, n\}$ (i.e., $a_{\sigma(i)}$ is the ith largest element in the collection $a_{1}, \ldots, a_{n}$ ) and the weight $\omega_{i}$ is defined as

$\omega_{i}=w^{*}\left(\sum_{j \leq i} p_{\sigma(j)}\right)-w^{*}\left(\sum_{j<i} p_{\sigma(j)}\right)$

with $w^{*}$ a monotone increasing function that interpolates the points $\left(i / n, \sum_{j \leq i} w_{j}\right)$ together with the point $(0,0) . w^{*}$ is required to be a straight line when the points can be interpolated in this way.

WOWA operators have already been used in previous works $[6,5]$ to aggregate information in $L$-fuzzy contexts. In this paper the generalization of WOWA operators to Choquet integrals [21] will allow to aggregate values taking into account the existing relations among them.

Given a fuzzy measure $[31,33]$ in $\mathcal{P}(X)$, the set of parts of $X$ :

Definition 3 A function $m: \mathcal{P}(X) \longrightarrow[0,1]$ is a fuzzy measure if and only if it satisfies the following axioms: (i) $m(\emptyset)=0$ and $m(X)=1$ (ii) monotonicity: $B_{1} \subseteq$ $B_{2} \subseteq X$ implies $m\left(B_{1}\right) \leq m\left(B_{2}\right)$.

Grabisch [25] reformulated the Choquet integral:

Definition 4 Given a fuzzy measure $m$ the Choquet integral with respect to $m$ can be expressed as:

$C h_{m}\left(a_{1} \ldots a_{N}\right)=\sum_{k=1}^{N} a_{\sigma(k)}\left(m\left(A_{\sigma(k)}\right)-m\left(A_{\sigma(k-1)}\right)\right)$

where $\{\sigma(1), \ldots \sigma(N)\}$ is a permutation of $\{1, \ldots, N\}$ such that $a_{\sigma(1)} \geq a_{\sigma(2)} \geq \cdots \geq a_{\sigma(N)}, A_{\sigma(k)}=\left\{a_{\sigma(j)} \mid j \leq\right.$ $k\}$ (therefore $A_{\sigma(r)}=\left\{a_{\sigma(1)}, \ldots, a_{\sigma(r)}\right\}$ when $r \geq 1$ and $A_{\sigma}(0)=\emptyset$.) 
¿From this definition we can see that weighted means, OWA and WOWA operators are special types of Choquet integrals [32].

\section{Proposition 1 Let $m$ be a fuzzy measure.}

(1) $C h_{m}$ is the weighted mean $M_{p}$ if $m(A)=\sum_{i \in A} p_{i}$, $\forall A \subseteq X, A \neq \emptyset$.

(2) $C h_{m}$ is the OWA operator $O_{W}$ if $m(A)=\sum_{i=1}^{|A|} w_{i}$, $\forall A \subseteq X, A \neq \emptyset$.

When the OWA operator is defined by means of a fuzzy quantifier, it is verified that for every monotonically increasing fuzzy quantifier $Q$, we have $O W A_{Q}=C h_{m}$ when $m$ is defined by $m(B)=Q(|B| /|X|), \forall B \subseteq X$.

(3) For every weighting vector $p$ and every regular monotonically non-decreasing fuzzy quantifier $Q$, we have $W O W A_{Q, p}=C h_{m}$ with $m$ defined by $m_{Q, p}=Q\left(\sum_{a_{i} \in B} p_{i}\right)$, for all $B \subseteq X$.

\section{$2.2 L$-fuzzy concept analysis}

In order to work with these $L$-fuzzy contexts, we have defined $[13,15,16,18]$ the derivation operators 1 and 2 given by means of these expressions:

$$
\forall A \in L^{X}, \forall B \in L^{Y}
$$

$$
\begin{aligned}
& A_{1}(y)=\inf _{x \in X}\{I(A(x), R(x, y))\} \\
& B_{2}(x)=\inf _{y \in Y}\{I(B(y), R(x, y))\}
\end{aligned}
$$

being $I$ a fuzzy implication operator defined in the lattice $(L, \leq)$.

Although any fuzzy implication operator can be used to define the derivation operators, we will use in this paper residuated implications and $L=[0,1]$.

The information stored in the context is visualized by means of the $L$-fuzzy concepts which are pairs $\left(A, A_{1}\right)$ $\in L^{X} \times L^{Y}$ with $A \in f i x(\varphi)$, set of fixed points of the operator $\varphi$, being defined from the derivation operators 1 and 2 as $\varphi(A)=\left(A_{1}\right)_{2}=A_{12}$. These pairs, whose first and second components are said to be the fuzzy extension and intension respectively, represent a group of objects that share a group of attributes.

Using the usual order relation between fuzzy sets, that is,

$\forall A, C \in L^{X}, \quad A \leq C \Longleftrightarrow A(x) \leq C(x) \quad \forall x \in X$,

we define the set $\mathcal{L}=\left\{\left(A, A_{1}\right) / A \in f i x(\varphi)\right\}$ with the order relation $\preceq$ defined as: $\forall\left(A, A_{1}\right),\left(C, C_{1}\right) \in$ $\mathcal{L},\left(A, A_{1}\right) \preceq\left(C, C_{1}\right)$ if $A \leq C\left(\right.$ or $\left.C_{1} \leq A_{1}\right)$.

We proved that $(\mathcal{L}, \preceq)$ is a complete lattice that is said to be the $L$-fuzzy concept lattice $[13,15]$.
On the other hand, given $A \in L^{X}$, (or $B \in L^{Y}$ ) we can obtain the associated $L$-fuzzy concept applying twice the derivation operators. In the case of using a residuated implication, the associated $L$-fuzzy concept is $\left(A_{12}, A_{1}\right)$ (or $\left.\left(B_{2}, B_{21}\right)\right)$.

Other important papers $[11,12,30]$ generalize the Formal Concepts Analysis using residuated implication operators. Moreover, there are extensions of Formal Concept Analysis to the interval-valued case [3,22,23] and to the fuzzy property-oriented and multi-adjoint concept lattices framework $[27,28]$.

We have also studied sequences of $L$-fuzzy contexts. A first study when $L=[0,1]$ was tackled in [4].

We begin by recalling the main definition:

Definition 5 An $L$-fuzzy context sequence is a sequence of tuples $\left(L, X, Y, R_{i}\right), i \in\{1, \ldots, n\}, n \in \mathbb{N}$, with $L$ a complete lattice, $X$ and $Y$ sets of objects and attributes respectively and $R_{i} \in L^{X \times Y}$, for all $i \in\{1, \ldots, n\}$, a family of $L$-fuzzy relations between $X$ and $Y$.

In order to summarize the information stored in the $L$-fuzzy context sequence, we defined the following relation $R_{F}$ using an OWA operator:

Definition 6 Let $\left(L, X, Y, R_{i}\right), i \in\{1, \ldots, n\}$ be the fuzzy context sequence and $F$ an OWA aggregation operator of dimension $n$. We can define an L-fuzzy relation $R_{F}$ that aggregates the information of the different Lfuzzy contexts, in the case that we want to study the largest values, by means of this expression:

$$
\begin{aligned}
R_{F}(x, y)= & F\left(R_{1}(x, y), R_{2}(x, y) \ldots R_{n}(x, y)\right)= \\
= & w_{1} \cdot b_{1}+w_{2} \cdot b_{2}+\cdots+w_{n} . b_{n}, \\
& \forall x \in X, y \in Y
\end{aligned}
$$

where $W=\left(w_{1}, w_{2}, \ldots w_{n}\right)$ is the weighting tuple associated with $F$ and $b_{i}$ the $i$ th largest element in the collection $R_{1}(x, y), R_{2}(x, y), \ldots R_{n}(x, y)$.

In [1] we have developed a general study of these $L$ fuzzy context sequences using $n$-ary OWA operators for a complete lattice $L$.

Moreover, the use of WOWA operators will allow to perform a proper treatment of the sequence better than the proposed previously. We defined in [6] an $L$-fuzzy relation $R_{F_{p w}}$ that aggregates the information of the different fuzzy contexts, by means of this expression:

Definition 7 Let $\left(L, X, Y, R_{i}\right), i=\{1, \ldots, n\}$ be the fuzzy context sequence and $F_{p w}$ an WOWA aggregation operator with $p=\left(p_{1}, p_{2}, \ldots p_{n}\right)$ and $w=\left(w_{1}, w_{2}, \ldots w_{n}\right)$ such that $p_{i}, w_{i} \in[0,1]$ and $\sum_{i} p_{i}=\sum_{i} w_{i}=1$. Then,

$$
\begin{aligned}
R_{F_{p w}}(x, y)= & F_{p w}\left(R_{1}(x, y), \ldots R_{n}(x, y)\right)= \\
& \sum_{i} \omega_{x y_{i}} R_{\sigma_{x y}(i)}(x, y)
\end{aligned}
$$


where for every $(x, y)$ we have $\sigma_{x y}=\left\{\sigma_{x y}(1), \ldots, \sigma_{x y}(n)\right\}$ a permutation of $\{1, \ldots, n\}$ such that $R_{\sigma_{x y}(i-1)}(x, y) \geq$ $R_{\sigma_{x y}(i)}(x, y)$ for all $i=\{2, \ldots, n\}$, and the weighting vector $\omega_{x y}$ defined in Definition 2 .

This relation $R_{F_{p w}}$ combines all the contexts of the sequence with two tables of weights. We considered interesting to use the weighting vector $p$ to give more relevance to the most recently contexts. On the other hand, we toke as a vector of weights $w$ one that will allow to highlight membership degrees close to 1 since we want to study the largest values.

Finally, as an important point in the study of $L$ fuzzy context sequences, in [7] we studied Temporal Trends defining Trend and Persistent Formal contexts.

\section{$2.3 \mathrm{~L}$-fuzzy contexts with multiple values}

In some cases, we have several values representing the relation between an object and an attribute. In the past [14] we defined the $L$-fuzzy contexts obtained from several expert opinions to study this situation. In that case multisets were used as a tool for working with the data without aggregating the observations:

Let $\mathrm{E}$ be a set, and let $\mathcal{M}(E)=\{f: E \longrightarrow \mathbb{N}\}$ be the multisets or bags of $\mathrm{E}$. Let $\mathrm{k} \in \mathbb{N}$ be a finite number; we denoted $M_{k}(E) \subseteq \mathcal{M}(E)$ to the set of multisets of cardinality $k$. We represented a multiset as a collection of elements of $E$ (with repetitions) that corresponds with the non null images of $E$ through $f$.

Definition 8 Let be $L=[0,1]$. An $M(L)$-fuzzy $k$ valued set $A$ associated to $X$ and an $M(L)$-fuzzy $k$ valued relation $R$ associated with $X$ and $Y$, are maps $A \in M_{k}(L)^{X}$ and $R \in M_{k}(L)^{X \times Y}$. We will represent the i-th observation of $R(x, y)$ by $R(x, y)_{i}$.

Using the theory of expertons [26], we defined an order relation $\leq$ in $M_{k}(L)$.

After that, we gave the following definition:

Definition 9 Let be $L=[0,1]$ and let $X$ and $Y$ be respectively the sets of objects and attributes, then the tuple $\left(M_{k}(L), X, Y, R\right)$ is said to be an $M(L)$-fuzzy $k$ valued context.

In the paper, the definition of the derivation operators 1 and 2 were adapted to the new situation.

Later [17], we analyzed the contexts with multiple weighted values where the set of experts $E$ and weights $P$ were introduced: $\left(M_{k}(L), X, Y, E, P, R\right)$.

In the first part of the paper, the $M(L)$-fuzzy $k$ valued concepts $(A, B)$ were studied using the theory of expertons:
Definition 10 An $M(L)$-fuzzy $k$-valued concept $(A, B)$ is a pair with $A \in M_{k}(L)^{X}$ and $B \in M_{k}(L)^{Y}$, verifying $A_{1}=B$ and $B_{2}=A$.

Also in this case, given $A \in M_{k}(L)^{X}$ (or $\left.B \in M_{k}(L)^{Y}\right)$, we can obtain the associated $M(L)$-fuzzy $k$-valued concept applying the derivation operators. In the case of using a residuated implication, the associated $M(L)$ fuzzy $k$-valued concept is $\left(A_{12}, A_{1}\right)$ (or $\left(B_{2}, B_{21}\right)$ ).

Recently [5], WOWA operators were used as aggregation tools in these contexts that represent the opinion of multiple experts.

In order to deepen in the study of the $L$-fuzzy contexts with multiple values, the rest of the paper is organized as follows: Section 3 proposes an algorithm for the study of multivalued contexts using Choquet integrals. In Section 4 we analyze these multivalued contexts as $L$-fuzzy context sequences. Section 5 illustrates these results by means of an example. Finally, conclusions and future work are detailed in Section 6.

\section{Aggregation of values in multivalued contexts by means of Choquet integrals}

The aim of this section is to improve the study of the contexts with multiple values.

We take as a departure point an $M(L)$-fuzzy $k$ valued context $\left(M_{k}(L), X, Y, R\right)$ with $|X|=n,|Y|=m$ and a set $E$ such that $|E|=k$. We want to analyze the information stored in this context.

In [5] we defined the Multivalued Aggregation Process (MAP) to analyze the multivalued contexts using aggregation operators. In the process, two options for the treatment of the data are described:

(1) Calculate the $M(L)$-Fuzzy $k$-valued concepts and aggregate the observations of the membership degrees of objects and attributes using OWA operators.

(2) Define a new relation $\bar{R}(x, y)$ aggregating the observations of the $M(L)$-Fuzzy $k$-valued context using WOWA operators $F_{p w}$ and later obtain the $L$-fuzzy concepts of the $L$-fuzzy context $(L, X, Y, \bar{R})$.

In that case, we considered the use of WOWA operators as a good option. However, there are some situations where the features of the data we are aggregating induce the use of other type of aggregations as Choquet integrals. We refer to situations where not only the individual observations, but also the groups or coalitions have to be taken into account.

As in the origin the $k$ observations were associated to a set $E$, from this point we will introduce this new set. We will start from the knowledge of the relationship 
among the elements of $E$ that will be represented by $Q \in L^{E \times E}$ such that $Q(x, y)$ measures the relationship between $x$ and $y$.

Starting from this relationship, we can define an $L$ fuzzy context $(L, E, E, Q)$ associated with $E$ and calculate the $L$-fuzzy concepts that are derived from certain special starting sets. These sets allow to establish solid relationships between groups of elements of the set $E$.

For every $e \in E$, let $\mathbf{e} \in L^{E}$ be such that $\mathbf{e}(e)=1$ and $\mathbf{e}(z)=0$, for any $z \in E, z \neq e$.

Definition 11 For every $e \in E$, if we consider $e$ as an object of the $L$-fuzzy context $(L, E, E, Q)$, then the pair $\mathcal{C}_{\mathbf{e}}=\left(\mathbf{e}_{12}, \mathbf{e}_{1}\right)$ is said to be the $L$-fuzzy concept derived from object $e$.

We can also consider $e$ as an attribute and we obtain $\left(\mathbf{e}_{2}, \mathbf{e}_{21}\right)$. It is immediate to prove the following proposition.

Proposition 2 If $R$ is a symmetric relation then $\mathcal{C}_{\mathbf{e}}=$ $\left(\mathbf{e}_{12}, \mathbf{e}_{1}\right)=\left(\mathbf{e}_{2}, \mathbf{e}_{21}\right)$.

These $L$-fuzzy concepts associated with every $e \in$ $E$ are useful for the definition of the measure in the Choquet integral that we will use.

Remark 1 If $R$ is not symmetric, the $L$-fuzzy concepts are not equal.

Henceforth, we are going to consider $e \in E$ as an object of the $L$-fuzzy context. For every $e, z \in E$, we denote $\mathbf{e}_{12}(z) \in L$ to the membership degree of object $z$ in the extension of the $L$-fuzzy concept $\mathcal{C}_{\mathbf{e}}$. Then, it is possible to define the $\alpha$-objects associated with $\mathcal{C}_{\mathbf{e}}$.

Definition 12 For every $\mathcal{C}_{\mathbf{e}}=\left(\mathbf{e}_{12}, \mathbf{e}_{1}\right)$ we define the set of $\alpha$-objects associated with $\mathcal{C}_{\mathbf{e}}$ as:

$T_{\mathcal{C}_{\mathbf{e}}}^{\alpha}=\left\{z \in E \mid \mathbf{e}_{12}(z) \geq \alpha\right\}$

At this time, we are able to define an algorithm Measure Choice Process (MCP) for establish the measure in the Choquet integral (see Algoritm 1).

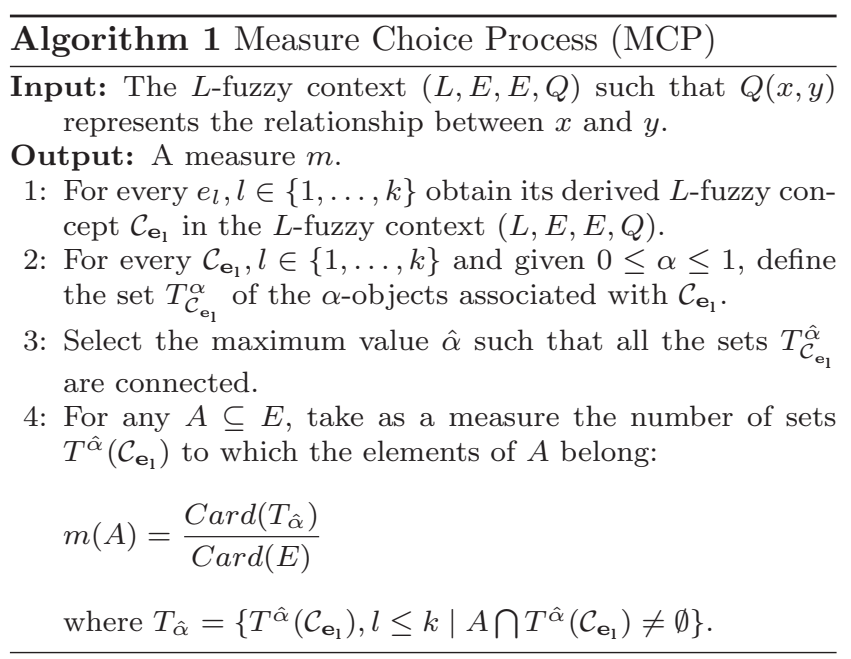

This measure gives more relevance to the criteria associated with the same $T_{\mathcal{C}_{\mathbf{e}_{1}}}^{\hat{\alpha}}$.

Definition 13 Given the previous fuzzy measure $m$, the Choquet integral with respect to $m$ can be expressed as:

$$
\begin{gathered}
C h_{m}\left(R(x, y)_{1}, R(x, y)_{2}, \ldots, R(x, y)_{k}\right)= \\
\sum_{l=1}^{k} R(x, y)_{\sigma(l)}\left(m\left(A_{\sigma(l)}\right)-m\left(A_{\sigma(l-1)}\right)\right)
\end{gathered}
$$

with $R(x, y)_{1}, R(x, y)_{2}, \ldots, R(x, y)_{k}$ the $k$ observations associated with the pair $(x, y),\{\sigma(1), \ldots \sigma(k)\}$ a permutation of $\{1, \ldots, k\}$ such that $R(x, y)_{\sigma(1)} \geq R(x, y)_{\sigma(2)} \geq$ $\cdots \geq R(x, y)_{\sigma(k)}$ and $A_{\sigma(l)}=\left\{e_{\sigma(j)} \mid j \leq l\right\}$. Therefore $A_{\sigma(r)}=\left\{e_{\sigma(1)}, \ldots, e_{\sigma(r)}\right\}$ when $r \geq 1$ and $A_{\sigma}(0)=\emptyset$.

Once defined the measure, we will analyze the characteristics of the observations that we want to aggregate and we will apply the algorithm Generalized Multivalued Aggregation Process (GMAP) (see Algorithm 2) in order to analyze the multivalued contexts by means of the aggregation processes.

\section{Multivalued contexts as sequences of contexts}

Sometimes the set of objects $X$ or attributes $Y$ in the $M(L)$-fuzzy $k$-valued context $\left(M_{k}(L), X, Y, R\right)$ represents a magnitude of time. In this section, our aim is the study of these situations using tools of $L$-fuzzy context sequences and maintaining the different observations of the $M(L)$-fuzzy $k$-valued context.

In [7], we define an $L$-fuzzy context sequence as a sequence of tuples $\left(L, X, Y, R_{i}\right), i \in\{1, \ldots, n\}, n \in$ $\mathbb{N}$, with $L$ a complete lattice, $X$ and $Y$ sets of objects and attributes and $R_{i} \in L^{X \times Y}$ for all $i \in\{1, \ldots, n\}$, a family of $L$-fuzzy relations between $X$ and $Y$. 


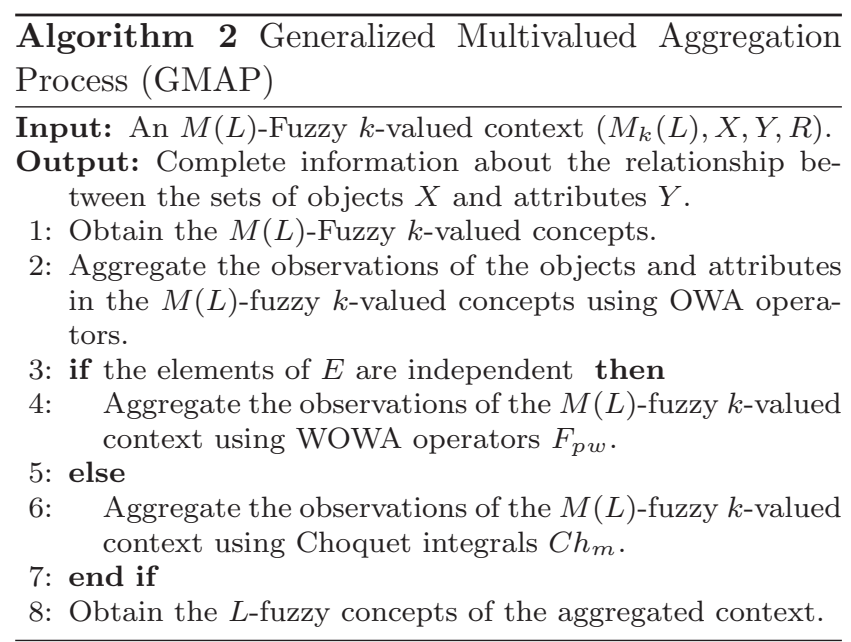

Suppose that $Y$ represents a magnitude of time. We can define an $L$-fuzzy context sequence for our $M(L)$ fuzzy $k$-valued context as follows:

Definition 14 Given an $M(L)$-fuzzy $k$-valued context $\left(M_{k}(L), X, Y, R\right)$ where $Y=\left\{y_{1}, y_{2}, \ldots y_{m}\right\}$ represents a magnitude of time and a set $E=\left\{e_{1}, \ldots, e_{k}\right\}$, we can define an $L$-fuzzy context sequence $\left(L, X, E, R_{j}\right)$, $j \in\{1, \ldots, m\}$ where, for all $j, R_{j} \in L^{X \times E}$ is the $L$ fuzzy relation given by:

$R_{j}\left(x, e_{l}\right)=R\left(x, y_{j}\right)_{l}, \forall x \in X, e_{l} \in E$.

A similar definition can be done if $X$ is the set that represents a magnitude of time:

Definition 15 Given an $M(L)$-fuzzy $k$-valued context $\left(M_{k}(L), X, Y, R\right)$ where $X=\left\{x_{1}, x_{2}, \ldots x_{n}\right\}$ represents a magnitude of time and a set $E=\left\{e_{1}, \ldots, e_{k}\right\}$, we can define an $L$-fuzzy context sequence $\left(L, E, Y, R_{i}\right)$, $i \in\{1, \ldots, n\}$ where, for all $i, R_{i} \in L^{E \times Y}$ is the $L$ fuzzy relation given by:

$R_{i}\left(e_{l}, y\right)=R\left(x_{i}, y\right)_{l}, \forall e_{l} \in E, y \in Y$.

Henceforth, we will assume that $Y$ is the set that changes in time. A parallel development could be performed for $X$ changing in time.

The study of temporal trends to identify the evolution in time of the $L$-fuzzy context sequence $\left(L, X, E, R_{j}\right)$, $j \in\{1, \ldots, m\}$ can be relevant in order to extract information from the $M(L)$-fuzzy $k$-valued context.

Our interest is focused on the study of the evolution of the relationship between the objects $X$ (or attributes $E$ ) with respect to one or several attributes $E$ (or objects $X)$.

For this purpose, we also use residuated implication operators in the $L$-fuzzy concepts associated with certain objects or attributes.
4.1 Trend and persistent objects and attributes

First, we study the evolution in time of an object or attribute obtaining its associated $L$-fuzzy concepts in the different $L$-fuzzy contexts of the sequence. ¿From this moment, we will denote by $\left(\underline{\mathcal{C}_{\mathbf{x}}^{j}}, \overline{\mathcal{C}_{\mathbf{x}}^{j}}\right)$ to the $L$-fuzzy concept associated with $\mathbf{x}$ in the $\bar{L}$-fuzzy context $\left(L, X, E, R_{j}\right)$, $j \in\{1, \ldots, m\}$.

Definition 16 Consider $x \in X, e \in E . \operatorname{Let} \mathcal{C}_{\mathbf{x}}^{j}=\left(\underline{\mathcal{C}_{\mathbf{x}}^{j}}, \overline{\mathcal{C}_{\mathbf{x}}^{j}}\right)$ and $\mathcal{C}_{\mathbf{e}}^{j}=\left(\mathcal{C}_{\mathbf{e}}^{j}, \overline{\mathcal{C}_{\mathbf{e}}^{j}}\right)$ the $L$-fuzzy concepts associated with $\mathbf{x}$ and $\mathbf{e}$ in the $L$-fuzzy context sequence $\left(L, X, E, R_{j}\right)$, $j \in\{1, \ldots, m\}$.

We define:

(i) $\operatorname{Trend}(x)=\left\{e \in E / \overline{\mathcal{C}_{\mathbf{x}}^{j}}(e) \leq \overline{\mathcal{C}_{\mathbf{x}}^{j+1}}(e)\right.$, for all $\left.j<m\right\}$

is the attribute set whose membership degrees in the different intensions of the $L$-fuzzy concepts $\left(\mathcal{C}_{\mathbf{x}}^{j}, \overline{\mathcal{C}_{\mathbf{x}}^{j}}\right)$ are non decreasing.

(ii) $\operatorname{Trend}(e)=\left\{x \in X / \mathcal{C}_{\mathbf{e}}^{j}(x) \leq \underline{\mathcal{C}_{\mathbf{e}}^{j+1}}(x)\right.$, for all $\left.j<m\right\}$ is the object set whose membership degrees in the different extensions of the $L$-fuzzy concepts $\left(\underline{\mathcal{C}_{\mathbf{e}}^{j}}, \overline{\mathcal{C}_{\mathbf{e}}^{j}}\right)$ are non decreasing.

We can say that they are the attributes that are more and more related to object $x$ and the objects more and more related to attribute $e$.

Moreover, it is easy to prove that $e \in \operatorname{Trend}(x) \Longleftrightarrow$ $x \in \operatorname{Trend}(e)$.

As the definition of Trend is very demanding, it can be interesting to relax the demand level and study Persistent objects and attributes.

Definition 17 Given $x \in X, e \in E$. Let $\mathcal{C}_{\mathbf{x}}^{j}=\left(\underline{\mathcal{C}_{\mathbf{x}}^{j}}, \overline{\mathcal{C}_{\mathbf{x}}^{j}}\right)$ and $\mathcal{C}_{\mathbf{e}}^{j}=\left(\underline{\mathcal{C}_{\mathbf{e}}^{j}}, \overline{\mathcal{C}_{\mathbf{e}}^{j}}\right)$ the $L$-fuzzy concepts associated with $\mathbf{x}$ and $\mathbf{e}$ in the $L$-fuzzy context sequence $\left(L, X, E, R_{j}\right)$, $j \in\{1, \ldots, m\}$.

(i) Persistent $(x)=\left\{e \in E / \overline{\mathcal{C}_{\mathbf{x}}^{j}}(e) \geq \overline{\mathcal{C}_{\mathbf{x}}^{1}}(e)\right.$, for all $\left.j<m\right\}$ is the set of attributes whose membership degrees in the fuzzy intensions of the $L$-fuzzy concepts $\left(\mathcal{C}_{\mathbf{x}}^{j}, \overline{\mathcal{C}_{\mathrm{x}}^{j}}\right)$ are bigger than or equal to the values of the $\bar{L}$-fuzzy concept $\left(\mathcal{C}_{\mathbf{x}}^{1}, \overline{\mathcal{C}_{\mathbf{x}}^{1}}\right)$.

(ii) Persistent $(e)=\left\{x \in X / \underline{\mathcal{C}_{\mathbf{e}}^{j}}(x) \geq \underline{\mathcal{C}_{\mathbf{e}}^{1}}(x)\right.$, for all $\left.j<m\right\}$ is the set of objects whose membership degrees in the fuzzy extensions of the $L$-fuzzy concepts $\left(\mathcal{C}_{\mathbf{e}}^{j}, \overline{\mathcal{C}_{\mathbf{e}}^{j}}\right)$ are bigger than or equal to the values of the $L$-fuzzy concept $\left(\underline{\mathcal{C}_{\mathbf{e}}^{1}}, \overline{\mathcal{C}_{\mathbf{e}}^{1}}\right)$.

The Trend and Persistent definitions set up pairs of objects and attributes for which the relationship is getting closer with the passage of time.

Following this idea, the study of tendencies of the $L$-fuzzy context sequence can be completed with the construction of binary Trend matrices. 
Definition 18 The Trend matrix $T M \subseteq X \times E$ is defined as:

$T M(x, e)= \begin{cases}1 & \text { if } e \in \operatorname{Trend}(x)(\text { equiv. } x \in \operatorname{Trend}(e)) \\ 0 & \text { in other case }\end{cases}$

We can consider now the formal context $(X, E, T M)$ and obtain its formal concepts to have a general idea of the trends in the relation between the objects $X$ and the attributes $E$.

These concepts represent groups of elements of $X$ and $E$ that are increasingly related to the passage of time. Therefore, they are robust relationships.

Definition 19 Consider the formal context $(X, E, T M)$ with $X$ the set of objects, $E$ the set of attributes and $T M \subseteq X \times E$. The formal concepts of $(X, E, T M)$ are called Trend formal concepts.

It is also possible to conduct a different study using Persistent definition:

Definition 20 The matrix $P M \subseteq X \times Y$ such that $P M(x, e)= \begin{cases}1 & \text { if } e \in P \text { Persistent }(x)(x \in P \text { ersistent }(e)) \\ 0 & \text { in other case }\end{cases}$ is called Persistent Matrix.

We can also consider $(X, E, P M)$ and calculate their formal concepts to obtain information about the tendencies between the objects of $X$ and the attributes of $E$. In this case it will be groups of objects and attributes that are more related than at the starting point of the study.

Definition 21 Consider the formal context $(X, E, P M)$. The formal concepts of $(X, E, P M)$ are called Persistent formal concepts.

As we have said before, the demand level is less in this case.

With the intention of completing the study, in the next section we are going to focus our attention on the elements of $E$.

\section{$4.2 L$-fuzzy context associated with an element of $E$}

Let $\left(M_{k}(L), X, Y, R\right)$ be an $M(L)$-fuzzy $k$-valued context where the observations are associated with a set $E$. Defining an $L$-fuzzy context for each element of $E$, we can perform an individualized study associated with each $e_{l} \in E$.

Definition 22 Given $e_{l} \in E$, the $L$-fuzzy context $\left(L, X, Y, R_{e_{l}}\right), l \in\{1 \ldots k\}$ where $R_{e_{l}}(x, y)=R(x, y)_{l}$ is said to be the $L$-fuzzy context associated with $e_{l}$.
If $Y$ is the set that represents time, the rows of each new relation will represent the evolution of the values associated with the elements of $X$ and those of $E$ in time.

Specifically, for every $y \in Y$ we have $\overline{\mathcal{C}_{\mathbf{y}}^{e}}(z)$ the membership degree of attribute $z$ in the intension of the $L$ fuzzy concept $\mathcal{C}_{\mathbf{y}}^{e}$. Then, for every $\alpha \leq 1$, the sets of $\alpha$-attributes associated with $\mathcal{C}_{\mathbf{y}}^{e}$ :

$T_{\mathcal{C}_{\mathbf{y}}^{e}}^{\alpha}=\left\{z \in Y \mid \overline{\mathcal{C}_{\mathbf{y}}^{e}}(z) \geq \alpha\right\}$

can be a good indicator of the relationship between different attributes according to the element $e$.

Of special interest are those $e \in E$ for which the value of the relationship $R_{e}(x, y)$ is increasing over time $Y$.

Definition 23 Given $e \in E$, we say that $e$ is a Leading element if for any $y_{i}, y_{j} \in Y, i \leq j$ then $R_{e}\left(x, y_{i}\right) \leq$ $R_{e}\left(x, y_{j}\right), \forall x \in X$.

The following property will demonstrate how the $L$ -fuzzy concepts of the contexts associated with these special elements $e \in E$ are.

Proposition 3 Let $e \in E$ be a Leading element. Let $\left(L, X, Y, R_{e}\right)$ be the $L$-fuzzy context associated with $e$, where $Y$ is a set that varies with time. For every $y \in Y$, let $\mathcal{C}_{\mathbf{y}}=\left(\mathcal{C}_{\mathbf{y}}, \overline{\mathcal{C}_{\mathbf{y}}}\right)$ be the L-fuzzy concept associated with $y \in Y$. It $\overline{i s}$ verified that:

(i) Given $y_{i}, y_{j} \in Y$, if $i \leq j$ then $\underline{\mathcal{C}_{\mathbf{y}_{\mathbf{i}}}}(x) \leq \underline{\mathcal{C}_{\mathbf{y}_{\mathbf{j}}}}(x), \forall x \in$ $X$.

(ii) Given $y_{i}, y_{j} \in Y$, if $i \leq j$ then $\overline{\mathcal{C}_{\mathbf{y}_{\mathbf{i}}}}(y) \geq \overline{\overline{\mathcal{C}_{\mathbf{j}}}}(y), \forall y \in$ $Y$.

(iii) Given $y_{i} \in Y, \overline{\mathcal{C}_{\mathbf{y}_{\mathbf{i}}}}(y)=1, \forall y \geq y_{i}$.

Proof

(i) $\mathcal{C}_{\mathbf{y}_{\mathbf{i}}}(x)=\left(\mathbf{y}_{\mathbf{i}}\right)_{2}(x)=R\left(x, y_{i}\right) \leq R\left(x, y_{j}\right)=\left(\mathbf{y}_{\mathbf{j}}\right)_{2}(x)=$ $\overline{\mathcal{C}_{\mathbf{y}_{\mathbf{j}}}}(x), \forall x \in X$.

(ii) $\overline{\overline{\mathcal{C}_{\mathbf{y}_{\mathbf{i}}}}}(y)=\left(\mathbf{y}_{\mathbf{i}}\right)_{21}(y)=\inf _{x \in X}\left(I\left(\left(\mathbf{y}_{\mathbf{i}}\right)_{2}(x), R(x, y)\right)\right)=$ $\inf _{x \in X}\left(I\left(R\left(x, y_{i}\right), R(x, y)\right)\right) \geq \inf _{x \in X}\left(I\left(R\left(x, y_{j}\right), R(x, y)\right)\right)=$ $\left(\mathbf{y}_{\mathbf{j}}\right)_{21}(y)=\overline{\mathcal{C}_{\mathbf{y}_{\mathbf{j}}}}(y), \forall y \in Y$.

(iii) Given $y_{i} \in Y$, and $y \geq y_{i}$, then $\overline{\mathcal{C}_{\mathbf{y}_{\mathbf{i}}}}(y)=\left(\mathbf{y}_{\mathbf{i}}\right)_{21}(y)=$ $\inf _{x \in X}\left(I\left(R\left(x, y_{i}\right), R(x, y)\right)\right) \geq \inf _{x \in X}(I(R(x, y), R(x, y)))=$ 1 . The last equality is a property of residuated implication operators.

Remark 2 By results (i) and (ii) of Proposition 3, if $i \leq j$ then $\mathcal{C}_{\mathbf{y}_{\mathbf{i}}} \succeq \mathcal{C}_{\mathbf{y}_{\mathbf{j}}}$.

Besides, we can prove the following result:

Proposition 4 Given $y_{i}, y_{j} \in Y$, with $i \leq j$, and $\alpha \leq$ 1 , then $T_{\mathcal{C}_{\mathbf{y}_{\mathbf{i}}}^{e}}^{\alpha} \supseteq T_{\mathcal{C}_{\mathbf{y}_{\mathbf{j}}}^{e}}^{\alpha}$. 
Proof We have to prove that if $y \in T_{\mathcal{C}_{\mathbf{y}_{\mathfrak{j}}}^{e}}^{\alpha}$ then $y \in T_{\mathcal{C}_{\mathbf{y}_{\mathrm{i}}}^{e}}^{\alpha}$.

As $y \in T_{\mathcal{C}_{\mathbf{y}_{\mathbf{j}}}^{e}}^{\alpha}$ if and only if $\overline{\mathcal{C}_{\mathbf{y}_{\mathbf{j}}}}(y) \geq \alpha$, and taking into account that $\overline{\mathcal{C}_{\mathbf{y}_{\mathbf{j}}}}(y) \leq \overline{\mathcal{C}_{\mathbf{y}_{\mathbf{i}}}}(y)$, then $\overline{\mathcal{C}_{\mathbf{y}_{\mathbf{i}}}}(y) \geq \alpha$. Thus, $y \in T_{\mathcal{C}_{\mathbf{y}_{\mathbf{i}}}^{e}}^{\alpha}$.

Therefore, as a particular case when $\alpha=1$, it is possible to represent the sets $T_{\mathcal{C}_{\mathrm{y}}^{e}}^{\alpha}, y \in Y$ as can be seen in Figure 1.

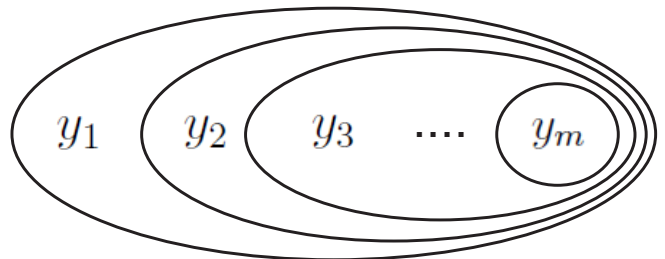

Fig. 1 Sets $T_{\mathcal{C}_{\mathrm{y}}^{e}}^{\alpha}, y \in Y$

We can perform a similar development if $X$ is the set that represents the time.

Now we can apply all these results to study a practical case where you can see its usefulness when we work with certain multivalued contexts.

\section{Practical application: Helping to evaluate improvement in learning processes}

We are interested in the study of the evolution of a group of $k$ students (set $E$ ) in the different subjects of which they are enrolled. To represent this situation we have an $M(L)$-fuzzy $k$-valued context $\left(M_{k}(L), X, Y, R\right)$ where $k=5, X$ is the set of subjects, $Y$ are the months the course lasts and $R \in M_{k}(L)^{X \times Y}$ associates each subject $x$ and every month $y$ the grades in $L=[0,1]$ obtained by the $k=5$ students (see Table 1 ).

Table 1 Relation $R$ of the $M(L)$-fuzzy $k$-valued context.

\begin{tabular}{|c|c|c|c|c|c|c|c|c|c|c|}
\hline$R$ & \multicolumn{5}{|c|}{$y_{1}$} & \multicolumn{5}{|c|}{$y_{2}$} \\
\hline$x_{1}$ & 0.1 & 0.5 & 1 & 1 & 0.7 & 0.1 & 0.6 & 1 & 0.9 & 0.6 \\
\hline$x_{2}$ & 0 & 0.3 & 0.9 & 1 & 0.5 & 0.2 & 0.5 & 1 & 1 & 0.4 \\
\hline$x_{3}$ & 0.2 & 0.6 & 0.8 & 0.8 & 0.3 & 0.3 & 0.6 & 1 & 0.9 & 0 \\
\hline$R$ & & & $y_{3}$ & & & & & $y_{4}$ & & \\
\hline$x_{1}$ & 0.2 & 0.7 & 1 & 1 & 0.5 & 0.3 & 0.8 & 1 & 1 & 0.3 \\
\hline$x_{2}$ & 0.4 & 0.7 & 0.9 & 1 & 0.4 & 0.2 & 0.9 & 0.9 & 0.8 & 0 \\
\hline$x_{3}$ & 0.1 & 0.6 & 1 & 0.8 & 0 & 0.3 & 0.6 & 1 & 1 & 0 \\
\hline
\end{tabular}

It has to keep in mind that class work is done in some cases in group and will be part of the student's grade, so the relationship among the students is important. This relationship $Q \in L^{E \times E}$ will be recorded in
Table 2 where $Q(x, y)$ is equal to 1 when both students collaborate together very positively and it is equal to 0 if the fact of being together is very negative for work.

Table 2 Relationship among the students.

\begin{tabular}{l|lllll}
\hline$Q$ & $e_{1}$ & $e_{2}$ & $e_{3}$ & $e_{4}$ & $e_{5}$ \\
\hline$e_{1}$ & 1 & 0.8 & 0 & 1 & 0 \\
$e_{2}$ & 0.8 & 1 & 0.6 & 0 & 0.8 \\
$e_{3}$ & 0 & 0.6 & 1 & 0.2 & 0.6 \\
$e_{4}$ & 1 & 0 & 0.2 & 1 & 0 \\
$e_{5}$ & 0 & 0.6 & 0.6 & 0 & 1 \\
\hline
\end{tabular}

Following Section 3 we can use GMAP (Algorithm 2) to obtain the general information of the $M(L)$-fuzzy $k$ valued context.

We consider in this case that the set $E$ is made up of dependent elements and then, we aggregate the observations using Choquet integrals $C h_{m}$ and later obtain the $L$-fuzzy concepts. To do this, we have to follow the algorithm Measure Choice Process (MCP).

The $L$-fuzzy concepts associated with the elements of $E$ have the extensions:

$$
\begin{aligned}
& \underline{\mathcal{C}_{\mathbf{e}_{1}}}=\left\{e_{1} / 1, e_{2} / 0, e_{3} / 0, e_{4} / 0.2, e_{5} / 0\right\} \\
& \overline{\mathcal{C}_{\mathbf{e}_{2}}}=\left\{e_{1} / 0.4, e_{2} / 1, e_{3} / 0.2, e_{4} / 0, e_{5} / 0.2\right\} \\
& \overline{\mathcal{C}_{\mathbf{e}_{3}}}=\left\{e_{1} / 0, e_{2} / 0.6, e_{3} / 1, e_{4} / 0.2, e_{5} / 0.6\right\} \\
& \overline{\mathcal{C}_{\mathbf{e}_{4}}}=\left\{e_{1} / 0.8, e_{2} / 0, e_{3} / 0, e_{4} / 1, e_{5} / 0\right\} \\
& \overline{\mathcal{C}_{\mathbf{e}_{5}}}=\left\{e_{1} / 0, e_{2} / 0.6, e_{3} / 0.6, e_{4} / 0, e_{5} / 1\right\}
\end{aligned}
$$

In this case, $\hat{\alpha}=0.4$ and the sets $T^{\hat{\alpha}}\left(\mathcal{C}_{\mathbf{e}_{1}}\right)$ are represented in Figure 2.

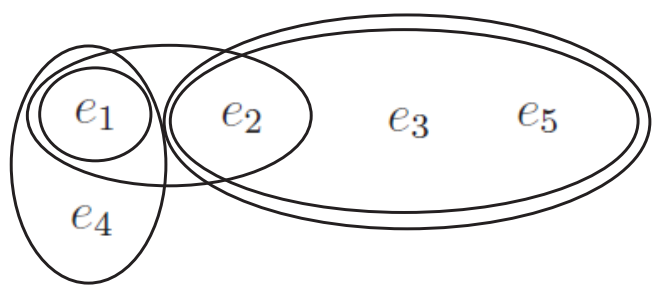

Fig. 2 Sets $T^{\hat{\alpha}}\left(\mathcal{C}_{\mathbf{e}_{1}}\right)$.

Then, we can take a measure $m$ following step 4 in MCP (see Algorithm 1).

For instance, we have:

$m\left(e_{1}\right)=m\left(e_{2}\right)=3 / 5, m\left(e_{3}\right)=m\left(e_{5}\right)=2 / 5, m\left(e_{4}\right)=1 / 5$

for the sets formed by a single element.

The aggregated relation (Definition 13) using a Choquet integral with this $m$ is shown in Table 3 .

We can obtain now the $L$-fuzzy concepts associated with the different attributes in order to study witch are the best grades for the group of students in the different 
Table 3 Aggregated relation $R_{C h_{m}}$.

\begin{tabular}{l|llll}
\hline$R_{C h_{m}}$ & $y_{1}$ & $y_{2}$ & $y_{3}$ & $y_{4}$ \\
\hline$x_{1}$ & 0.72 & 0.72 & 0.78 & 0.72 \\
$x_{2}$ & 0.62 & 0.74 & 0.78 & 0.60 \\
$x_{3}$ & 0.64 & 0.76 & 0.66 & 0.78 \\
\hline
\end{tabular}

months of the period. The extensions of these $L$-fuzzy concepts are:

$\underline{\mathcal{C}_{\mathbf{y}_{1}}}=\left\{x_{1} / 0.72, x_{2} / 0.62, x_{3} / 0.64\right\}$

$\underline{\mathcal{C}_{\mathbf{y}_{2}}}=\left\{x_{1} / 0.72, x_{2} / 0.74, x_{3} / 0.76\right\}$

$\underline{\mathcal{C}_{\mathbf{y}_{3}}}=\left\{x_{1} / 0.78, x_{2} / 0.78, x_{3} / 0.66\right\}$

$\underline{\mathcal{C}_{\mathbf{y}_{4}}}=\left\{x_{1} / 0.72, x_{2} / 0.60, x_{3} / 0.78\right\}$

Therefore, we can conclude that using the relations between the students with Choquet integrals, the general results can be summarized in this way:

(i) In the first month $\left(y_{1}\right)$ the qualification of subject $x_{1}$ stands out.

(ii) However, in the second and fourth month $\left(y_{2}, y_{4}\right)$ the grade of subject $x_{3}$ is the one that highlights.

(iii) Finally, in the third month $\left(y_{3}\right)$ subjects $x_{1}$ and $x_{2}$ correspond to the best grades.

Also, as $Y$ represents the evolution in time in this case, we can interpret our $M(L)$-fuzzy $k$-valued context as a sequence of $L$-fuzzy contexts. Therefore, we can also apply Section 4 and calculate the Trend and Persistent formal concepts.

In this case, we have the matrix $T M$ (Def. 18) in Table 4.

Table 4 Matrix $T M$.

\begin{tabular}{l|lllll}
\hline$T M$ & $e_{1}$ & $e_{2}$ & $e_{3}$ & $e_{4}$ & $e_{5}$ \\
\hline$x_{1}$ & 1 & 1 & 1 & 0 & 0 \\
$x_{2}$ & 0 & 1 & 0 & 0 & 0 \\
$x_{3}$ & 0 & 1 & 1 & 0 & 0 \\
\hline
\end{tabular}

We can consider now the formal context $(X, E, T M)$ and obtain its formal concepts to have a general idea of the trends between the objects $X$ and the attributes $E$. This is the result:

$$
(\emptyset, E),\left(\left\{x_{1}\right\},\left\{e_{1}, e_{2}, e_{3}\right\}\right),\left(\left\{x_{1}, x_{3}\right\},\left\{e_{2}, e_{3}\right\}\right),\left(X,\left\{e_{2}\right\}\right)
$$

The Trend formal concepts represent groups of subjects in which a group of students has obtained an increasingly better result throughout the course.
For instance, for the second concept we can conclude that students $e_{1}, e_{2}$ and $e_{3}$ have improved their results in subject $x_{1}$ every month.

Besides, in this practical case the Persistent matrix $P M$ (Def. 20) can be seen in Table 5.

Table 5 Matrix $P M$.

\begin{tabular}{l|lllll}
\hline$P M$ & $e_{1}$ & $e_{2}$ & $e_{3}$ & $e_{4}$ & $e_{5}$ \\
\hline$x_{1}$ & 1 & 1 & 1 & 0 & 0 \\
$x_{2}$ & 1 & 1 & 1 & 0 & 0 \\
$x_{3}$ & 0 & 1 & 1 & 1 & 0 \\
\hline
\end{tabular}

Their Persistent formal concepts represent groups of subjects in which a group of students has obtained a better result than at the beginning of the course. This is the result for our example:

$(\emptyset, E),\left(\left\{x_{1}, x_{2}\right\},\left\{e_{1}, e_{2}, e_{3}\right\}\right),\left(\left\{x_{3}\right\},\left\{e_{2}, e_{3}, e_{4}\right\}\right),\left(X,\left\{e_{2}, e_{3}\right\}\right)$

For instance, for the second concept we can conclude that students $e_{1}, e_{2}$ and $e_{3}$ have improved their results in $x_{1}$ and $x_{2}$.

Finally, we can perform a differentiated study for every student $e \in E$ following section 4 . We have studied the different $L$-fuzzy contexts associated with each student. For instance, for student $e_{2}$ we have the $L$-fuzzy context associated with $R_{e_{2}}$ (see Table 6).

Table $6 L$-fuzzy context associated with student $e_{2}$.

\begin{tabular}{l|llll}
\hline$R_{e_{2}}$ & $y_{1}$ & $y_{2}$ & $y_{3}$ & $y_{4}$ \\
\hline$x_{1}$ & 0.5 & 0.6 & 0.7 & 0.8 \\
$x_{2}$ & 0.3 & 0.5 & 0.7 & 0.9 \\
$x_{3}$ & 0.6 & 0.6 & 0.6 & 0.6 \\
\hline
\end{tabular}

We have checked that $e_{2}$ is the only Leading student in this practical case. This student is the one who has got increasing results in all the subjects during the analyzed period of time.

For this student, we obtain the final evaluation of each subject $x_{i} \in X$, aggregating the corresponding results of the different months in $R_{e_{2}}$.

In this case, we have used WOWA operators with $p=(1 / 10,2 / 10,3 / 10,4 / 10)$ and $w=(4 / 10,3 / 10,2 / 10,1 / 10)$ since we want to give more relevance to the highest and closest in time values.

Then, the aggregated values for $x_{1}, x_{2}$ and $x_{3}$ are $0.74,0.78$ and 0.6 respectively. So, we can order the subjects from best to worst for $e_{2}$ depending on the positive evolution over time:

$x_{2} \succ x_{1} \succ x_{3}$ 


\section{Conclusions and future work}

The possibility of applying Choquet integrals in some specific situations in $M(L)$-fuzzy $k$-valued contexts allows aggregating elements in a more coherent way. These situations are represented by contexts in which there is a dependency among the different observations corresponding to an object and an attribute. We have proposed an algorithm to be applied in these cases defining previously a measure based on Choquet integrals.

Besides, when one of the sets represents time, we can use tools of contexts that evolve in time obtaining more solid conclusions from our $M(L)$-fuzzy $k$-valued contexts. Specifically, we have obtained robust relationships between the set of objects and attributes over time analyzing the formal concepts of the tendency matrices.

In the future we will compare these results with those obtained using other measures such as those derived from overlap indexes. The use of penalty functions will also provide us a good option to study differences among diverse aggregation functions.

\section{Acknowledgements}

This paper is partially supported by the Research Group "Intelligent Systems and Energy (SI+E)" of the University of the Basque Country - UPV/EHU, under Grant GIU 16/54 and by the Research Group "Artificial Intelligence and Approximate Reasoning" of the Public University of Navarra, under TIN2016-77356-P (MINECO, AEI/FEDER, UE).

\section{Compliance with ethical standards}

Conflict of interest The authors declare that they have no conflict of interest regarding the publication of this article.

Ethical approval This article does not contain any studies with human participants or animals performed by any of the authors.

\section{References}

1. C. Alcalde, A. Burusco: L-fuzzy context sequences on complete lattices, IPMU 2014, Lecture Notes in Computer Science Part III, CCIS 444, pp. 31-40, 2014.

2. C. Alcalde, A. Burusco: The use of two relations in $L$ fuzzy contexts, Information Sciences 301 (1), pp. 1-12, 2015.

3. C. Alcalde, A. Burusco, R. Fuentes-González, I. Zubia: Treatment of L-fuzzy contexts with absent values, Information Sciences 179 (1-2), pp. 1-15, 2009.
4. C. Alcalde, A. Burusco, R. Fuentes-González: The study of fuzzy context sequences International Journal of Computational Intelligence Systems 6 (3), pp. 518-529, 2013.

5. C. Alcalde, A. Burusco: Multivalued contexts associated with criteria, International Journal of General Systems 47 (2), pp. 118-136, 2018.

6. C. Alcalde, A. Burusco: WOWA operators in fuzzy context sequences, 16th World Congress of the International-Fuzzy-Systems-Association (IFSA) / 9th Conference of the European-Society-for-Fuzzy-Logicand-Technology, EUSFLAT 2015, Gijón, Spain. In: Advances in Intelligent Systems Research 89, pp. 357-362, 2015.

7. C. Alcalde, A. Burusco, H. Bustince, A. Jurío, J.A. Sanz: Evolution in time of the $L$-fuzzy context sequences. Information Sciences 326, pp. 202-214, 2016.

8. S. Al-Janabi and E. Alwan: Soft Mathematical System to Solve Black Box Problem through Development the FARB Based on Hyperbolic and Polynomial Functions, IEEE 10th International Conference on Developments in eSystems Engineering (DeSE), Paris, pp. 3742, 2017.

9. S. Al-Janabi, I. Al-Shourbaji, M. A. Salman: Assessing the suitability of soft computing approaches for forest fires prediction, Applied Computing and Informatics 14 (2), pp. 214-224, 2018.

10. S. Al-Janabi, S. Rawat, A. Patel, I. Al-Shourbaji: Design and evaluation of a hybrid system for detection and prediction of faults in electrical transformers, International Journal of Electrical Power and Energy Systems 67, pp. 324-335, 2015

11. R. Bělohlávek: Fuzzy Galois Connections, Math. Logic Quarterly 45 (4), pp. 497-504, 1999.

12. R. Bělohlávek, V. Vychodil: What is a fuzzy concept lattice. In Proceedings of the Third International Conference on Concept Lattice and their Applications, CLA 2005 Olomounc, Czech Republic, pp. 34-45, 2005.

13. A. Burusco, R. Fuentes-González: The Study of the Lfuzzy Concept Lattice, Mathware and Soft Computing 1 (3), pp. 209-218, 1994.

14. A. Burusco, R. Fuentes-González: Contexts obtained from several expert opinions. Sixth IEEE International Conference on Fuzzy Systems, Barcelona pp. 339-342, 1997.

15. A. Burusco, R. Fuentes-González: Construction of the Lfuzzy Concept Lattice, Fuzzy Sets and Systems 97 (1), pp. 109-114, 1998.

16. A. Burusco, R. Fuentes-González: Concept lattices defined from implication operators, Fuzzy Sets and Systems 114 (1), pp. 431-436, 2000.

17. A. Burusco, R. Fuentes-González: Contexts with multiple weighted values, The International Journal of Uncertainty, Fuzziness and Knowledge-based Systems 9 (3), pp. 355-368, 2001.

18. A. Burusco, R. Fuentes-González: The study of the interval-valued contexts, Fuzzy Sets and Systems 121, pp. 69-82, 2001.

19. T. Calvo, R. Mesiar: Weighted triangular norms-based aggregation operators, Fuzzy Sets and Systems 137, pp. $3-10,2003$

20. T. Calvo, R. Mesiar: Aggregation operators: ordering and bounds, Fuzzy Sets and Systems 139, pp. 685-697, 2003.

21. G. Choquet: Theory of capacities. Annales de l'Institut Fourier 5, pp.131-295, 1953.

22. Y. Djouadi, H. Prade: Interval-Valued Fuzzy Galois Connections: Algebraic Requirements and Concept Lattice Construction, Fundamenta Informaticae 99 (2), pp. 169$186,2010$. 
23. Y. Djouadi, H. Prade: Possibility- theoretic extension of derivation operators in formal concept analysis over fuzzy lattices, FODM 10 (4), pp. 287-309, 2011.

24. B. Ganter, R. Wille: Formal concept analysis: Mathematical foundations, Springer, Berlin - New York, 1999.

25. M. Grabisch: Fuzzy integral in multicriteria decision making, Fuzzy Sets and Systems 69 (3), pp. 279-298, 1995.

26. A. Kaufmann: Theory of expertons and fuzzy logic. Fuzzy Sets and Systems 28, pp. 295-304, 1988.

27. J. Medina, M. Ojeda-Aciego: Multi-adjoint t-concept lattices, Information Sciences 180 (5), pp. 712-725, 2010.

28. J. Medina, M. Ojeda-Aciego: Dual multi-adjoint concept lattices, Information Sciences 225, pp. 47-54, 2013.

29. A. Patel, S. Al-Janabi, I. AlShourbaji, J. Pedersen: A novel methodology towards a trusted environment in mashup web applications, Computers and Security 49, pp. 107-122, 2015.

30. S. Pollandt: Fuzzy Begriffe Springer, 1997.

31. M. Sugeno: Theory of Fuzzy Integrals and its Applications, (PhD Dissertation). Tokyo Institute of Technology, Tokyo, Japan, 1974

32. V. Torra: The weighted OWA Operator, International Journal of Intelligent Systems 12, pp. 153-166, 1997.

33. Z. Wang, G.J. Klir: Fuzzy Measure Theory, Plenum Press, New York and London, 1992.

34. R. Wille: Restructuring lattice theory: an approach based on hierarchies of concepts, in: Rival I. (Ed.), Ordered Sets, Reidel, Dordrecht-Boston, pp. 445-470, 1982.

35. R.R. Yager: On ordered weighted averaging aggregation operators in multi-criteria decision making, IEEE Transactions on Systems, Man and Cibernetics 18, pp. 183$190,1988$. 